\title{
NextGen Oncology Education in the Digital Era
}

\author{
Renata Zahu ${ }^{1}$
}

${ }^{1}$ Department of Radiation Oncology, Amethyst Radiotherapy Center, Cluj, Romania

Have you ever thought about how much time you spend on social media? If you are further adding Netflix et al., probably a guilty feeling might grip your stomach. Would it be possible to spend your online time in a more useful way? At the beginning of each year we make promises and we set goals. Most of them are health-related: we are going to work out more, reduce carbohydrates in our diet, cut down on coffee or Coke, but of course by midspring we just give up. I propose to challenge yourself in a different way this year and stay Oncoalert (1).

The easiest way is to look for websites and journals which offer a daily or weekly cover-up on what is new in Oncology. One of my favourite is PracticeUpdate ${ }^{\circledR}$ (2), with expert curated content based on one's field of practice and optimized for display on any device. The platform offers information on new clinical trials, recently published abstracts and journal scans, flagging those that are critical to practitioners. They also present expert opinion, conference coverages and educational videos. A detailed analysis of the practice-changing RAPIDO trial was already available on the platform when all we had was just the abstract for the American Society of Clinical Oncology (ASCO) 2020 International Conference (3). You can opt to receive a daily, weekly or monthly newsletter with all the latest news that best suit your field of interest.

A similar digital newspaper is The ASCO Post (4), developed in partnership with the American Society of Clinical Oncology. Each issue features coverage of clinical cancer research, policy news, patient care, clinical practice issues, and thoughtful commentary by leaders in the field of clinical oncology. They offer an evening news e-mail, which might be a great way to end a rather long day (and dream about successful treatments). Or, you might want to keep the evenings for yourself and read it in the morning-beware that burnout haunts our practice. Since these platforms are more like gateways, do not forget to check the real, peer-reviewed 
material and judge the information by yourself.

The ability to filter different opinions on various Medical and Radiation Oncology topics is key when navigating the mednet project, (5), started by Nadine Housri MD when her father was diagnosed with cancer and when she subsequently reached out to her robust network for help and advice. It is a wonderful platform to share experience and learn from experts to enrich your practice and get the best treatment for your patients. One asks a question on a practical issue that is not covered by the guidelines, e.g. what dose constraint should be used for the heart when treating mediastinal lymphoma, and somebody out there, more experienced, will answer based on his/her practice. Referents come from prestigious oncology centres in the US and are usually experts in their field (Dr Perez is also there, preaching from his lifelong experience in Radiation Oncology). So when you have a difficult case, type your question in the evening and if you are lucky enough, while you are sleeping, somebody from a different GMT time zone will answer it for you by the morning.

Speaking about events that happen overnight, do you tweet? If not, the time has come. On the bright side of the platform there are plenty of societies and experts in our field who are sharing their projects and latest research results. You can join interesting discussions in the comment section. Since this is easy reading, you can do this while commuting or waiting in line at the supermarket. This is how I found out that 30 Gy definitive radiotherapy might someday be just enough to cure selected HPV+-ve oropharyngeal cancer patients (6). Be aware that these results have to be further validated in larger, phase III trials to become practicechanging. In this respect, the account radoncreview_org (7) is a fantastic source of info and a gateway to probably the most comprehensive resource in our field-the evergreen book on evidence-based Radiation Oncology (8). Constraints are hard to catch but you will find them all collected there.

Of course, social networking could also be a great way to build connections with your colleagues and foster collaborations or enrich your knowledge, but it is also a valuable tool to be closer to your patients. Being a member of a journal club, a scientific community or a patient advocacy group is a good way to increase your motivation and get inspired (9). Seeing a picture on Facebook of your 70 years-old sarcoma patient skiing down the mountain can be the positive feedback you need to rest assured that working hard helps others in their pursuit of happiness.

\section{Abbreviations:}

ASCO - American Society of Clinical Oncology 


\section{Statements:}

\section{Conflict of interest: None}

\section{Funding: None}

\section{References:}

1. Oncoalert [account on Instagram]. USA: Instagram; 2020 [updated 2021]. Available from: https://www.instagram.com/oncoalert/

2. PracticeUpdate [internet]. USA: Elsevier Inc; 2013 [updated 2021]. Available from: https://www.practice update.com/explore/

3. Bahadoer RR, Dijkstra EA, van Etten B, et al. Short-course radiotherapy followed by chemotherapy before total mesorectal excision (TME) versus preoperative chemoradiotherapy, TME, and optional adjuvant chemotherapy in locally advanced rectal cancer (RAPIDO): a randomised, open-label, phase 3 trial. Lancet Oncol. 2021 Jan;22(1):29-42.

4. The ASCO Post [internet]. USA; 2010 [updated 2021]. Available from: https://ascopost.com/

5. theMedNet [internet]. USA; 2014 [updated 2021] Available from: https://www.themednet.org/home/index

6. Riaz N, Sherman E, Pei X, et al. Precision Radiotherapy: Reduction in Radiation for Oropharyngeal Cancer in the 30 ROC Trial. J Natl Cancer Inst. 2021 Jan 12:djaa184.

7. @radoncreview [Internet]. USA: Twitter; 2019 [updated 2021]. Available from: https://twitter.com/ radoncreview

8. RadOncReview [internet]. USA; 2019 [updated 2021]. Available from: https://www.radoncreview.org/

9. Sedrak MS, Attai DJ, George K, et al. Integrating Social Media in Modern Oncology Practice and Research. Am Soc Clin Oncol Educ Book. 2018 May 23;38:894-902. 